\title{
Inversion of spherical means using geometric inversion and Radon transforms
}

\author{
A E Yagle \\ Department of Electrical Engineering and Computer Science, The University of \\ Michigan, Ann Arbor, MI 48109-2122, USA
}

Received 8 May 1991, in final form 23 June 1992

\begin{abstract}
We consider the problem of reconstructing a continuous function on $\mathcal{R}^{n}$ from certain values of its spherical means. A novel aspect of our approach is the use of geometric inversion to recast the inverse spherical mean problem as an inverse Radon transform problem. We define two spherical mean inverse problems: the entire problem and the causal problem. We then present a dual filtered backprojection algorithm for the entire problem and an invariant imbedding algorithm for the causal problem. We then show how geometric inversion can be used to transform the entire and causal problems into complete and exterior inverse Radon transform problems, respectively. We also consider the uniqueness problem, for which we prove a sufficiency theorem and we note an application of these results to diffraction tomography.
\end{abstract}

\section{Introduction}

\subsection{The basic problem}

We consider the following problem. Given values of the 'spherical means'

$$
\hat{f}(t, e)=\int_{|x|=t e \cdot e_{x}} f(x) \mathrm{d} \sigma=\left.\int_{S} f\left(|x|, e_{x}\right) \frac{|x|^{n-1}}{e \cdot e_{x}}\right|_{|x|=t e \cdot e_{x}} \mathrm{~d} e_{x}
$$

of a function $f(x)=f\left(|x|, e_{x}\right)$ defined on $x \in \mathcal{R}^{n}$, determine $f(x)$ from $\hat{f}(t, e)$. Here $e$ and $e_{x}$ are unit vectors in $\mathcal{R}^{n}, \mathrm{~d} \sigma=|x|^{n-1} /\left(e \cdot e_{x}\right) \mathrm{d} e_{x}$ is differential surface area on the surface of the sphere $|x|=t e \cdot e_{x}$ in $\mathcal{R}^{n}$, and $S$ is the unit half-sphere $e \cdot e_{x} \geqslant 0$ in $\mathcal{R}^{n}$ (although the orientation of $S$ actually depends on $e$, this will not be explicitly indicated). Note that $|x|=t e \cdot e_{x}$ describes a sphere in $\mathcal{R}^{n}$ that passes through the origin, has diameter $t$ and centre at the point $t e / 2$ in $\mathcal{R}^{n}$. Also note that if $f(x)$ is a continuous function, $\hat{f}(t, e)$ will also be a continuous function in both $t$ and $e$.

This problem of inverting spherical means has been treated by many authors, e.g. [1,2]. However, the usual problem is to recover $f(x)$ from its integrals over spheres whose centres all lie on a plane; only the even part of $f(x)$ with respect to this plane can be recovered. Nonetheless, this problem is useful in reconstructing a scattering acoustic medium from its responses to an impulsive point source located at various places on its surface; in the Born approximation these responses are simply the spherical means of the medium reflectivity $[3,4]$. 
The problem we investigate is to recover $f(x)$ from its integrals over spheres passing through the origin. This problem is of interest in diffraction tomography; we present a novel application of the results of this paper to diffraction tomography in subsection 1.3 below. The novelty of our approach is that we use geometric inversion to transform the inverse problem (1.1) into an inverse Radon transform problem. By reflecting functions across a given sphere, integrals over spheres are transformed into integrals over planes.

Specifically, we define two spherical mean inverse problems: the entire problem of reconstructing $f(x)$ from $\{\hat{f}(t, e)\}$ for all $t$ and $e$; and the causal problem of reconstructing $\{f(x),|x|<t\}$ from $\{\hat{f}(\tau, e),|\tau|<t\}$ for any $t>0$. We then present a dual filtered backprojection algorithm for the entire problem, and an invariant imbedding algorithm for the causal problem. We then show how geometric inversion can be used to transform the entire and causal problems into complete and exterior inverse Radon transform problems, respectively. Finally, we state and prove a sufficiency theorem on the inversion of (1.1).

\subsection{Review of diffraction tomography}

In this section we quickly review the basic result of diffraction tomography. This allows us to develop in subsection 1.3 a new application of the spherical mean inverse problem. The material in subsection 1.2 is taken from [5].

The basic two-dimensional (2D) diffraction tomography problem is formulated as follows. A 2D acoustic medium has constant wave speed $c_{0}$, except in a region

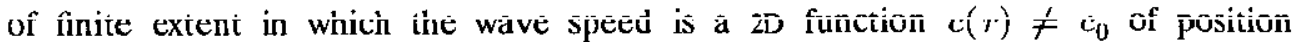
$r=(x, y) \in \mathcal{R}^{2}$. An infinite plane wave $u_{0}\left(t-s_{0} \cdot r / c_{0}\right)$, propagating in the direction specified by the unit vector $s_{0}=\left(\cos \phi_{0}, \sin \phi_{0}\right)$, is used to probe the inhomogeneous region. The scattered field $d\left(r, t ; s_{0}\right)$ is measured for all time $t$ by an array of sensors along the line $x=l_{0}$. The goal is to reconstruct $c(r)$ from the measurements $d\left(r=\left(l_{0}, y\right), t ; s_{0}\right)$ of the scattered field, for all positions $y$ along the line of sensors, time $t$, and directions of incidence $s_{0}$. Note that the problem is overdetermined we are using data with three degrees of freedom to reconstruct a function with two degrees of freedom. The problem is illustrated in figure 1.

This problem is of interest in offset vertical seismic profiling and ultrasound medical tomography. In both cases, the wavelengths of the incident wave field $u_{0}(t)$ and scattered wave field $d\left(r, t ; s_{0}\right)$ are long enough such that diffraction effects cannot be neglected.

First, define the scattering potential

$$
o(r)=1-\left(c_{0} / c(r)\right)^{2} .
$$

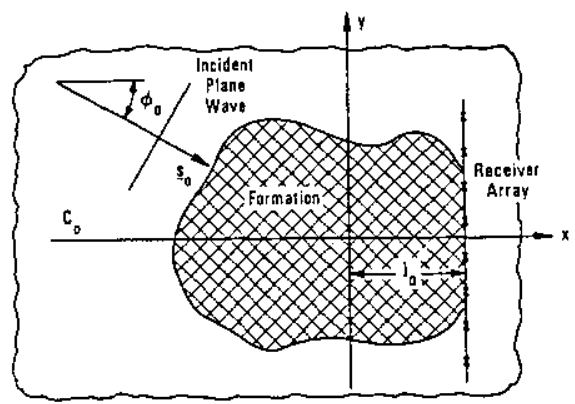

Figure 1. The basic diffraction tomography problem with a fixed array. 


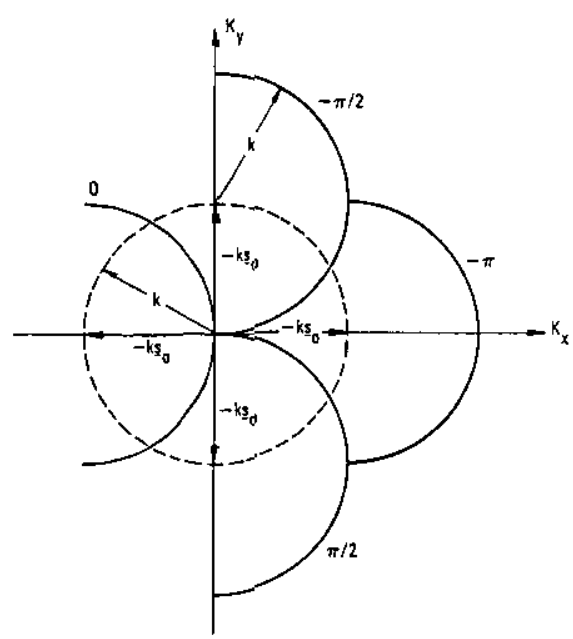

Figure 2. Some of the semi-circles on which the spatial Fourier transform $O(K)$ of scattering potential is reconstructed in the diffraction tomography problem.

Note that $o(r)=0$ outside the inhomogeneous region. Then the temporal Fourier transform $U\left(r, k ; s_{0}\right)$ of the total wave ficld satisfies

$$
\left(\nabla^{2}+k^{2}\right) U\left(r, k ; s_{0}\right)=k^{2} o(r) U\left(r, k ; s_{0}\right)
$$

where $k=\omega / c_{0}$ is the wavenumber.

Under the assumption that $o(r)$ is small (weak scattering), we may use either the Born or Rytov approximations. Define the temporal Fourier transforms of the incident and scattered wave fields as $U_{0}(k)$ and $D\left(r, k ; s_{0}\right)$, where

$$
D\left(r, k ; s_{0}\right)=\frac{2 \mathrm{i}}{k^{2}} \begin{cases}U\left(r, k ; s_{0}\right) / U_{0}(k)-\mathrm{e}^{\mathrm{i} k s_{0} \cdot r} & \text { Born } \\ \left(\log \left[U\left(r, k ; s_{0}\right) / U_{0}(k)\right]-\mathrm{i} k s_{0} \cdot r\right) \mathrm{e}^{\mathrm{i} k s_{0} \cdot r} & \text { Rytov. }\end{cases}
$$

Also define the 2D spatial Fourier transform of the scattering potential as $O(K)=\mathcal{F F}_{r \rightarrow K}\{o(r)\}$, and the 1D spatial Fourier transform of the measurements $D\left(r, k ; s_{0}\right)$ along the array of sensors $r=\left(l_{0}, y\right)$ as

$$
\tilde{D}\left(k^{\prime}, k ; s_{0}\right)=\int_{-\infty}^{\infty} D\left(r=\left(l_{0}, y\right), k ; s_{0}\right) \mathrm{e}^{\mathrm{i} k^{\prime} y} \mathrm{~d} y .
$$

Then it is straightforward to show (see [5]) that the known $\bar{D}\left(k^{\prime}, k ; s_{0}\right)$ and the unknown $O(K)$ are related by

$$
\bar{D}\left(k^{\prime}, k ; s_{0}\right)=\frac{\mathrm{e}^{\mathrm{i} \sqrt{k^{2}-k^{\prime 2}} l_{0}}}{\sqrt{k^{2}-k^{\prime 2}}} O\left(K=k\left(s-s_{0}\right)\right)
$$

where the unit vector $s$ is defined as

$$
s=(\cos \theta, \sin \theta) \quad \sin \theta=k^{\prime} / k \quad \cos \theta=\sqrt{1-\left(k^{\prime} / k\right)^{2}} \quad|\theta|<\pi / 2 .
$$

Equation (1.6) shows that the measurements $d\left(r=\left(l_{0}, y\right), t ; s_{0}\right)$ of the scattered field specify the Fourier transform $O(K)$ of the scattering potential on semi-circles of radius $k$ centred on $-k s_{0}=\left(-k \cos \phi_{0},-k \sin \phi_{0}\right)$, where $\phi_{0}$ is the angle of incidence of the probing plane wave (see figure 1). This is illustrated in figure 2 for $\phi_{0}=0, \pi / 2, \pi$ and $3 \pi / 2$. 
Three comments are in order here. First, why do we get semi-circles instead of full circles? This occurs because evanescent waves (waves which decay exponentially with distance instead of propagating) cannot be measured in practice. This restricts $\left|k^{\prime}\right|<k$ (see [5]), which from (1.7) restricts $|\theta|<\pi / 2$. Second, in the high-frequency limit $k \rightarrow \infty$ the semi-circles become lines, so the diffraction tomography problem becomes an inverse Radon transform problem, and (1.6) becomes the projection-slice theorem. Third, it is clear that without loss of generality we may set $l_{0}=0$; the only occurrence of $l_{0}$ is in the factor $\mathrm{e}^{\mathrm{i} \sqrt{k^{2}-k^{\prime \prime}} l_{0}}$ in (1.6), which we recognize as the migration operator between the lines $x=l_{0}$ and $x=0$.

\subsection{A new application of the spherical mean inverse problem in diffraction tomography}

In this section we show, for the first time, that measurements along an array of sensors are not necessary. In fact, measurements at a single sensor are sufficient to reconstruct the scattering potential in either the Born or Rytov approximations, provided either: (1) truly wideband data are available; or (2) the average value of the potential is known. We show this by formulating this problem as the spherical mean inverse problem considered in the rest of the current paper.

The problem we consider in this section is identical to the problem considered in subsection 1.2, except that now the scattered field $d\left(r, t ; s_{0}\right)$ is measured only at the origin $r=(0,0)$, instead of along the line $\left\{r=\left(l_{0}, y\right),-\infty<y<\infty\right\}$. Clearly there is no loss of generality in taking the origin to be the site of measurements. From (1.5), this amounts to measuring

$$
D\left((0,0), k ; s_{0}\right)=\frac{1}{2 \pi} \int_{-k}^{k} \tilde{D}\left(k^{\prime}, k ; s_{0}\right) \mathrm{d} k^{\prime}
$$

since $\bar{D}\left(k^{\prime}, k ; s_{0}\right)$ for $\left|k^{\prime}\right|>k$ are the evanescent components of the scattered field, which are negligible. Inserting (1.6) in (1.8), setting $l_{0}=0$, and changing variables from $k^{\prime}$ to $\theta$ using (1.7) (the denominator $\sqrt{k^{2}-k^{\prime 2}}$ in (1.6) disappears in the variable change) shows that the measurements are actually

$$
\begin{aligned}
D\left((0,0), k ; s_{0}\right) & =\frac{1}{2 \pi} \int_{-\pi / 2}^{\pi / 2} O\left(K=k\left(\cos \theta-\cos \phi_{0}, \sin \theta-\sin \phi_{0}\right)\right) \mathrm{d} \theta \\
& =\frac{1}{2 \pi} \int_{-\pi / 2}^{\pi / 2} O\left(|K|=2 k \sin \left(\frac{\theta-\phi_{0}}{2}\right)\right) \mathrm{d} \theta \\
& =\frac{1}{2 \pi} \mathcal{B}\{O(K)\}(t, \theta) \quad|\theta|<\pi / 2 .
\end{aligned}
$$

The backprojection operator $\mathcal{B}\{\cdot\}$ is defined in (2.2) below; it is essentially the spherical mean operator. The last equality in (1.9) comes from noting that the semi-circle $K^{\prime}=k\left(s-s_{0}\right)$ has diameter $2 k$ and is centred at $-k s_{0}=$ $\left(-k \cos \phi_{0},-k \sin \phi_{0}\right)$. Changing $\phi_{0}$ to $\phi_{0}+\pi$ transforms the sine in the third term of (1.9) into a cosine.

Equation (1.9) states that $D\left((0,0), k ; s_{0}\right)$ are essentially the semi-circular means of $O(K)$; this is evident from (1.8), which integrates along each of the semi-circles in figure 2 . To transform the semi-circular means into full-circular means we need the 'other halves' of the semi-circles in figure 2 . 
To obtain these 'other halves,' we simply note that since $o(r)$ is real, $O(-K)=$ $O^{*}(K)$, where * denotes complex conjugate. Since the integral of complex conjugates is the complex conjugate of the integral, the semi-circular means of $O(-K)$ will be the complex conjugates of the semi-circular means of $O(K)$. But the right-semicircular means of $O(-K)$ are the same as the left-semi-circular means of $O(K)$. Therefore, a complete set of full-circular means of $O(K)$ can be obtained by simply adding $D\left((0,0), k ; s_{0}\right)$ and $D^{*}\left((0,0), k ;-s_{0}\right)$, for each $k$ and $s_{0}$.

We can then reconstruct $O(K)$, and then scattering potential $o(r)$ and wave speed $c(r)$, from these circular means using the methods proposed in the rest of this paper. If truly wideband $(0<k<\infty)$ data are available, we may use the procedure of subsection 2.3 below. Otherwise, we must know $O(K)$ for small $|K|$; this low-wavenumber information corresponds roughly to the average value of $o(r)$.

The new result of this section is not surprising, from a degrees-of-freedom point of view. $o(r)$ has two degrees of freedom, while the measurements $d\left(r=\left(l_{0}, y\right), t ; s_{0}\right)$ in subsection 1.2 have three degrees of freedom, so clearly there is some redundancy. This section has shown how this redundancy can be removed.

\subsection{Geometric inversion}

Let $r e$ be a point in $\mathcal{R}^{n}$ located a distance $r$ from the origin in the direction specified by the unit vector $e$. The geometric inversion of $r e$ across the sphere of radius $T$ in $\mathcal{R}^{n}$ is the point $(T / r) e$.

Consider the sphere $|x|=t e \cdot e_{x}$ in $\mathcal{R}^{n}$, which passes through the origin and has diameter $t$ and centre at the point $t e / 2$. The geometric inversion of this sphere across the sphere of radius $T$ in $\mathcal{R}^{n}$ is $T /|x|=t e \cdot e_{x}$, which can be rewritten as $e \cdot x=T / t$, which is a hyperplane passing within a distance $T / t$ of the origin and having a normal unit vector $e$.

This can be seen more clearly in $\mathcal{R}^{2}$. Let $(r, \theta)$ and $(x, y)$ be the polar and rectangular coordinates, respectively, of a point in $\mathcal{R}^{2}$. The geometric inversion of the circle $r=t \cos \theta$ (which passes through the origin and has diameter $t$ ) across the circle $r=T$ is $T / r=t \cos \theta$, which can be rewritten as $x=r \cos \theta=T / t$, which is a line perpendicular to the $x$-axis. Other circles passing through the origin can be similarly handled by first rotating coordinates.

\section{Radon transforms, backprojection and causality}

\subsection{Causality properties of transforms}

Recall that the 'Radon transform' of $f(x), x \in \mathcal{R}^{n}$ is defined as

$\mathcal{R}\{f(x)\}(t, e)=\int_{0}^{\infty} \int_{S} f\left(|x|, e_{x}\right) \delta\left(t-|x| e \cdot e_{x}\right)|x|^{n-1} \mathrm{~d} e_{x} \mathrm{~d}|x|$

where $e$ is a unit vector in $\mathcal{R}^{n}$. Note that $(t, e)$ should not be regarded as a point in $\mathcal{R}^{n}$, since $\mathcal{R}\{f(x)\}\left(0, e_{1}\right) \neq \mathcal{R}\{f(x)\}\left(0, e_{2}\right)$ if $e_{1} \neq e_{2}$; however, it is true that $\mathcal{R}\{f(x)\}(t, e)=\mathcal{R}\{f(x)\}(-t,-e)$.

Since the Radon transform of a function is its integral over the planes $t=e \cdot x$, the Radon transform has the following anti-causality property: For any $T>0$, $\mathcal{R}\{f(x)\}(T, e)$ depends only on the values $\{f(x),|x| \geqslant T\}$. This is clear since the minimum distance between the origin and the plane $T=e \cdot x$ is $T$. 
The 'backprojection transform' of $f(x), x \in \mathcal{R}^{n}$ is defined as

$$
\mathcal{B}\{f(x)\}(t, e)=\int_{S} f\left(|x|=t e \cdot e_{x}, e_{x}\right) \mathrm{d} e_{x}
$$

where $e$ and $e_{x}$ are unit vectors in $\mathcal{R}^{n} . \mathcal{B}$ is half the adjoint operator to $\mathcal{R}$ ([7], $\mathrm{p}$ 115). The half is due to the integration over the half-sphere $S$; since $(|x|, e)$ and $(-|x|,-e)$ represent the same point in $\mathcal{R}^{n}, f(|x|, e)=f(-|x|,-e)$, and the integral over the opposing half-sphere equals the integral over $S$

$$
\begin{aligned}
\int_{e \cdot e_{x}<0} f\left(|x|=t e \cdot e_{x}, e_{x}\right) \mathrm{d} e_{x} \\
=\int_{e \cdot e_{x}>0} f\left(|x|=-t e \cdot e_{x},-e_{x}\right) \mathrm{d} e_{x}=\int_{e \cdot e_{x}>0} f\left(|x|=t e \cdot e_{x}, e_{x}\right) \mathrm{d} e_{x} .
\end{aligned}
$$

More importantly, we have from (1.1) that

$$
\hat{f}(t, e)=\left.\int_{S} f\left(|x|, e_{x}\right) \frac{|x|^{n-1}}{e \cdot e_{x}}\right|_{|x|=t e \cdot e_{x}} \mathrm{~d} e_{x}=t \mathcal{B}\left\{|x|^{n-2} f(x)\right\}
$$

so that inverting the spherical mcan operator (1.1) is essentially equivalent to inverting the backprojection operator (2.2). Indeed, we have

$$
f(x)=\frac{1}{|x|^{n-2}} \mathcal{B}^{-1}\left\{\frac{1}{i} \hat{f}(t, e)\right\}=\mathcal{B}^{-1}\left\{\frac{1}{i} \hat{f}(t, e)\right\} \quad \text { if } n=2 .
$$

Therefore, in the following, any results on inverting the backprojection operator will be directly applicable to inverting the spherical mean operator.

A simple example illustrating the difference between the two operators is in order here. Let $n=3$ and $f(x)=1$ everywhere in $\mathcal{R}^{3}$. Then $\mathcal{B}\{f(x)\}=\int_{S} \mathrm{~d} e_{x}=2 \pi$ (the surface area of the half-sphere $S$ ) and $\hat{f}(t, e)=4 \pi(t / 2)^{2}=\pi t^{2}$ (the surface area of a sphere with radius $t / 2$ ). For this example (2.3) becomes

$$
\hat{f}(t, e)=t \mathcal{B}\{|x|\}=t \int_{S} t e \cdot e_{x} \mathrm{~d} e_{x}=t^{2} \int_{0}^{2 \pi} \int_{0}^{\pi / 2} \cos \theta \sin \theta \mathrm{d} \theta \mathrm{d} \phi=\pi t^{2}
$$

where 0 is polar angle measured from direction $e, e \cdot e_{x}=\cos \theta$, and $\mathrm{d} e_{x}=$ $\sin \theta \mathrm{d} \theta \mathrm{d} \phi$.

Inversion of both operators will be aided by the following causality property: For any $T>0, \hat{f}(T, e)$ depends only on the values $\{f(x),|x| \leqslant T\}$. This is clear since the maximum distance between the origin and the sphere $|x|=T e \cdot e_{x}$ is $T$. Another way to see this is to note that backprojection at the point $(T, e)$ can also be viewed as the average over all planes (in $(t, e))|x|=t e \cdot e_{x}$ passing through $(T, e)$; any such plane must pass closer to the origin than $T$, so that for any such plane the minimum distance from the origin is $|x|<T$.

We show in section 3 that $\{f(x),|x|<T\}$ may be reconstructed from $\{\hat{f}(t, e), t<T\}$, provided that $f(x)$ is known in an arbitrarily small sphere centred at the origin; this is the causal spherical mean inverse problem. However, the availability of the additional data $\{\hat{f}(t, e), t>T\}$ will be shown to alter the poorlyconditioned causal spherical mean inverse problem into the relatively well-conditioned entire spherical mean inverse problem. 


\subsection{The exterior Radon transform problem}

The anti-causality property of the Radon transform implies that it should be possible to reconstruct $\{f(x),|x|>t\}$ from its Radon transform values $\{\mathcal{R}\{f(x)\}(\tau, e),|\tau|>t\}$, for any $t>0$. This is the exterior Radon transform problem. For $n=2$ it may be solved as follows [6].

Assume $f(x)$ has finite support, so that there exists $T$ such that $f(x)=0$ for all $|x|>T$. Then $\mathcal{R}\{f(x)\}(\tau, e)=0$ for $|\tau|>T$. Let $t$ be any number such that $0<t<T$. Both the unknown function $f(x)=f(|x|, \theta)$ and its known Radon transform $\mathcal{R}\{f(x)\}(\tau, \phi)$ (the projection angle $\phi$ replaces the unit vector $e=(\cos \phi, \sin \phi)$ for $n=2)$ are periodic in their angular variables $\theta$ and $\phi$, respectively. Hence both may be expanded in Fourier series in their angular variables, yielding

$$
\begin{aligned}
& f(x)=f(|x|, \theta)=\sum_{m=-\infty}^{\infty} F_{m}(|x|) \mathrm{e}^{\mathrm{i} m \theta} \\
& \mathcal{R}\{f(x)\}(\tau, \phi)=\sum_{m=-\infty}^{\infty} R F_{m}(\tau) \mathrm{e}^{\mathrm{i} m \phi}
\end{aligned}
$$

where

$$
\begin{aligned}
& F_{m}(|x|)=\frac{1}{2 \pi} \int_{0}^{2 \pi} f(|x|, \theta) \mathrm{e}^{-\mathrm{i} m \theta} \mathrm{d} \theta \\
& R F_{m}(\tau)=\frac{1}{2 \pi} \int_{0}^{2 \pi} \mathcal{R}\{f(x)\}(\tau, \phi) \mathrm{e}^{-\mathrm{i} m \phi} \mathrm{d} \phi .
\end{aligned}
$$

Then the unknown function harmonics $\left\{F_{m}(|x|), t<|x|<T\right\}$ may be computed from the known projection harmonics $\left\{R F_{m}(\tau), t<\tau<T\right\}$ using the formula [6]

$$
F_{m}(|x|)=-\frac{1}{\pi} \int_{|x|}^{T} \frac{\frac{\mathrm{d}}{\mathrm{d} \tau} R F_{m}(\tau) P_{m}(\tau /|x|)|x|}{\sqrt{\tau^{2}-|x|^{2}}} \mathrm{~d} \tau .
$$

where $P_{m}(\tau)=\cos \left(m \cos ^{-1} \tau\right)$ is the Chebyschev polynomial of the first kind of order $m$.

It is clear from (2.8) that $\{f(x),|x|>t\}$ can be reconstructed in principle from $\{\mathcal{R}\{f(x)\}(\tau, \phi),|\tau|>t\}$. However, there are practical difficulties in using (2.8), since $P_{m}(\tau) \simeq(1 / 2)(2 \tau)^{m}$ for large $\tau$ and $m$, so that evaluation of the integral (2.8) will be numerically unstable for $|x| \rightarrow 0$ or $m \rightarrow \infty$. Hence the exterior Radon transform problem is ill-posed, and it must be regularized. One way to do this follows.

First, we must assume that the function $f(x)$ is angularly bandlimited, so that its Fourier expansion (2.6a) has only a finite number of terms. This amounts to assuming $F_{m}(|x|)=0$ for $|m|>M$, for some $M$. The Radon transform $\mathcal{R}\{\dot{f}(x)\}$ will then also be angularly bandlimited, so that $R F_{m}(\tau)=0$ for $|m|>M$. Then (2.8) need only be applied for the $2 M+1$ values of $m$ such that $|m| \leqslant M$. Since only a finite number of projection angles will be available in practice, this assumption is not unreasonable.

Second, we must accept that we cannot completely reconstruct $f(x)$ from $\mathcal{R}\{f(x)\}$ using (2.8); rather, we can only reconstruct $\{f(x),|x|>t\}$ from 
$\{\mathcal{R}\{f(x)\}(\tau, \phi),|\tau|>t\}$ for some $t>0$ (note that this is the problem stated at the beginning of this section). Then (2.8) need only be applied for $|x|>t>0$. The worst case of evaluating $P_{m}(\tau /|x|)$ in $(2.8)$ is $m=M, \tau=T$ and $|x| \rightarrow t$; if $M$ is large and $t$ is small relative to $T$, then $P_{m}(\tau /|x|) \simeq(1 / 2)(2 T / t)^{M}<\infty$. Note that even this regularized problem is very poorly conditioned.

This regularization is significant to the results of this paper, since this regularized exterior Radon transform problem (with $t=1$ ) will be applied to causal inversion of spherical means in subsection 3.4 below.

\subsection{Dual filtered hackprojection algorithm for the entire spherical mean inverse problem}

The transforms $\mathcal{R}$ and $\mathcal{B}$ are related by [7]

$$
f(x)=C_{n} \mathcal{B}_{(t, e) \rightarrow\left(|x|, e_{x}\right)}\left\{\left(\mathcal{H} \frac{\mathrm{d}}{\mathrm{d} t}\right)^{n-1} \mathcal{R}_{\left(|x|, e_{x}\right) \rightarrow(t, e)}\{f(x)\}\right\}
$$

where $\mathcal{H}$ denotes the Hilbert transform $\mathcal{H}\{f(t)\}=f(t) * \frac{-1}{\pi t}$ and $C_{n}=(2 \pi)^{1-n}$ (recall that, in the definition (2.2) of $\mathcal{B}, S$ is the unit half-sphere). Discretizing this leads to the well-known technique of 'filtered backprojection' and it immediately suggests the following algorithm for inverting (1.1).

For the spherical mean problem, both $f(x)$ and its spherical means $\hat{f}(t, e)$ are continuous functions. Thus we may interchange $\left(|x|, e_{x}\right)$ and $(t, e)$ in $(2.9)$, and replace $f(x)$ with $\hat{f}(t, e)$. Then, given the spherical means $\{\hat{f}(t, e)\}$ defined in $(1.1)$ for all $(t, e), f(x)$ may be recovered from $\{\hat{f}(t, e)\}$ using

$$
\begin{aligned}
f\left(|x|, e_{x}\right) & =\frac{1}{|x|^{n-2}} \mathcal{B}_{(t, e) \rightarrow\left(|x|, e_{x}\right)}^{-1}\left\{\frac{\hat{f}(t, e)}{t}\right\} \\
& =\left(\frac{C_{n}}{|x|^{n-2}}\right)\left(\mathcal{H} \frac{\mathrm{d}}{\mathrm{d}|x|}\right)^{n-1} \mathcal{R}_{(t, e) \rightarrow\left(|x|, e_{x}\right)}\left\{\frac{\hat{f}(t, e)}{t}\right\} .
\end{aligned}
$$

In words: Since the composition of the Radon transform, the derivative-Hilbert transform and backprojection is the identity, the inverse of the last operator is the composition of the first two operators.

\subsection{Discussion}

The above algorithm has two major advantages. First, it is relatively simpie, in that it requires only a forward Radon transform and a derivative-Hibbert transform operation; the latter can be implemented in the same way that it is implemented in filtered backprojection [7]. Sccond, the entire spherical mean inverse problem, which this algorithm solves, is relatively well-conditioned compared to the causal spherical mean inverse problem considered in section 3 .

However, this algorithm also has a major disadvantage, in that it requires knowledge of $\{\hat{f}(t, e)\}$ for all $t$ in order to recover $f(x)$ for any $x$. This follows since the Radon transform $\mathcal{R}_{(t, e)-\left(|x|, e_{\mathbf{x}}\right)}$ requires $\{\hat{f}(t, e)\}$ for all $t$ (in particular, for arbitrarily large $t$ ). Note that even if $f(x)$ has compact support, its spherical means $\{\hat{f}(t, e)\}$ will not have compact support, since even for arbitrarily large $t$ the sphere $|x|=t e \cdot e_{x}$ will intersect the support of $f(x)$. Thus $\{\hat{f}(t, e)\}$ cannot be windowed arbitrarily to zero for large $t$. 
Applying this procedure to the diffraction tomography problem of subsection 1.3 , we have the amusing result that a wide-band diffraction tomography problem may be solved by Radon transforming the temporal Fourier transform of measurements at a single point, and then applying the derivative-Hilbert transform operator to obtain the scattering potential in the spatial Fourier domain! However, the data must be truly wide-band $(0<k<\infty)$, for the reason noted above.

\section{Causal inversion of spherical means}

Since the spherical mean is a causal operator, it should be possible to causally invert it. In order to take advantage of this causality, recall that we defined the causal inverse spherical mean problem as follows. Let $T>0$. Given the values $\{\hat{f}(\tau, e), 0 \leqslant \tau \leqslant T\}$, determine $\{f(x), 0 \leqslant|x| \leqslant T\}$.

We present two different algorithms for solving this problem. One algorithm uses an invariant imbedding approach. The other uses geometric inversion to transform the problem into the exterior Radon transform problem. The second algorithm can also be used to solve the entire spherical mean inverse problem (see subsection 3.6); this shows the versatility of the geometric inversion approach.

\subsection{Invariant imbedding algorithm}

From (1.1) it is clear that $\hat{f}(t, e)$ depends on $\left\{f\left(|x|, e_{x}\right), 0 \leqslant|x| \leqslant t\right\}$. Suppose we increment the size of the problem from $t$ to $t+\Delta$. This introduces an additional 'shell' of data $\{\hat{f}(t+\Delta, e), e \in S\}$, and an additional 'shell' of unknowns $\left\{f\left(|x|+\Delta, e_{x}\right), e_{x} \in S\right\}$. The additional data can then be used to determine the additional unknowns (in conjunction with previous data). Thus $f(x)$ can be recursively reconstructed in increasing $|x|$.

To show how this works, we specialize to the 2D problem; the extension to an arbitrary number of dimensions is straightforward. Let the radius $|x|$ of $x$ in $f(x)=f(|x|, \theta)$ and the radius $t$ of its 2D spherical mean $\hat{f}(t, \phi)$ both be discretized to integer multiples of a (small) discretization length $\Delta$. The portion of the circle $|x|=t \cos (\theta-\phi)$ that intersects the annulus $t<|x|<t+\Delta$ subtends an angle $2 \Delta \theta=2 \cos ^{-1}[t /(t+\Delta)]$. Assume that $f(x)=f(|x|, \theta)$ does not vary significantly in the thin sector $\{(t, \theta): t<|x|<t+\Delta, \phi-\Delta \theta<\theta<\phi+\Delta \theta\}$.

Now suppose we have reconstructed $\{f(x), 0 \leqslant|x| \leqslant t\}$, and we wish to extend the region in which $f(x)$ has been reconstructed from $0 \leqslant|x| \leqslant t$ to $0 \leqslant|x| \leqslant t+\Delta$. For each $0 \leqslant \phi<2 \pi$ we have

$$
\begin{aligned}
\hat{f}(t+\Delta, \phi)= & \int_{\phi-\pi / 2}^{\phi+\pi / 2} f(|x|=(t+\Delta) \cos (\theta-\phi), \theta) \mathrm{d} \theta \\
= & \left\{\int_{\phi-\pi / 2}^{\phi-\Delta \theta}+\int_{\phi+\Delta \theta}^{\phi+\pi / 2}\right\} f(|x|=(t+\Delta) \cos (\theta-\phi), \theta) \mathrm{d} \theta \\
& +\int_{\phi-\Delta \theta}^{\phi+\Delta \theta} f(|x|=(t+\Delta) \cos (\theta-\phi), \theta) \mathrm{d} \theta \\
= & \left\{\int_{\phi-\pi / 2}^{\phi-\Delta \theta}+\int_{\phi+\Delta \theta}^{\phi+\pi / 2}\right\} f(|x|=(t+\Delta) \cos (\theta-\phi), \theta) \mathrm{d} \theta \\
& +f(|x|=t+\Delta, \phi) 2 \Delta \theta .
\end{aligned}
$$


The first integral in the last equality depends only on values of $f(x)$ for which $0 \leqslant|x| \leqslant t$. Since these values of $f(x)$ have already been reconstructed, the first integral may be computed from them. The 2D spherical mean $\hat{f}(t+\Delta, \phi)$ is known, so (3.1) immediately determines $f(|x|=t+\Delta, \phi)$. Using (3.1) in this way for each $0 \leqslant \phi<2 \pi$, we have extended the region in which $f(x)$ has been reconstructed from $0 \leqslant|x| \leqslant t$ to $0 \leqslant|x| \leqslant t+\Delta$.

We can then use $\hat{f}(t+2 \Delta, \phi)$ to extend the region in which $f(x)$ has been reconstructed from $0 \leqslant|x| \leqslant t+\Delta$ to $0 \leqslant|x| \leqslant t+2 \Delta$. Continuing in this way, we can reconstruct $\{f(x), 0 \leqslant|x| \leqslant T\}$ from $\{\hat{f}(\tau, e), 0 \leqslant \tau \leqslant T\}$, provided that we already know $f(x)$ in a small circle centred at the origin, namely $\{f(x), 0 \leqslant|x| \leqslant \epsilon\}$. This is necessary to initialize the above recursion. In higher dimensions, the arc $\phi-\Delta \theta<\theta<\phi+\Delta \theta$ becomes a cone $\left|e \cdot e_{x}\right| \leqslant t /(t+\Delta)$; if $\Delta$ is small, this cone will also be small.

\subsection{Numerical example of invariant imbedding algorithm}

We demonstrate the above procedure with a simple example, which is meant to be illustrative only. Consider the circularly symmetric 'mountain' function $f(|x|, \theta)=$ $\mathrm{e}^{-|x|},|x| \geqslant 0$ in $\mathcal{R}^{2}$. Its $2 \mathrm{D}$ spherical means (which are actually circular means) are also circularly symmetric, and a little algebra shows them to be

$$
\begin{aligned}
\hat{f}(t, \phi)= & \hat{f}(t, 0)=\int_{-\pi / 2}^{\pi / 2} t \mathrm{e}^{-t \cos \theta} \mathrm{d} \theta=2 t \int_{0}^{\pi / 2} \mathrm{e}^{-t \cos \theta} \mathrm{d} \theta \\
& =\pi t\left(I_{0}(t)-L_{0}(t)\right)
\end{aligned}
$$

where $I_{0}(t)$ is the modified Bessel function of the first kind of order zero and $L_{0}(t)$ is the modified Struve function of order zero. The integral definitions of $I_{0}(t)$ and $L_{0}(t)$, given in (9.6.16) and (12.2.2) of [8], respectively, were used to derive (3.2).

Before procceding with the example, two comments are in order, based on the observation $\left((12.2 .6)\right.$ of [8]) that $\pi t\left(I_{0}(t)-L_{0}(t)\right) \simeq 2$ for large $t$. Firstly, even though $f(x)$ decays exponentially to zero with increasing $|x|$, its spherical means do not decay to zero at all. This is an example of how spherical means should not be windowed to zero for large $t$. Secondly, in the limit $t \rightarrow \infty$, the circle $|x|=t \cos \theta$ becomes a line through the origin, and

$$
\hat{f}(t, \phi) \simeq \int_{-\infty}^{\infty} \mathrm{e}^{-|r|} \mathrm{d} r=2
$$

in agreement with the asymptotic behaviour of $\pi t\left(I_{0}(t)-L_{0}(t)\right)$.

We chose $T=1$ and $\Delta=1 / N$. The spherical means (3.2) were computed using Simpson's rule for numerical integration at $N+1$ points in the interval $0 \leqslant \theta \leqslant \pi / 2$. The imbedding equation (3.1) was implemented numerically by breaking up the integral over previously-reconstructed values of $f(x)$ into integrals over the annuli $\{i \Delta<|x|<(i+1) \Delta, 1 \leqslant i \leqslant N\}$; in each annulus $f(x)$ was assumed to be constant. This yiclded the recursive equation

$$
\begin{aligned}
f((n+1) \Delta) & \left(\hat{f}((n+1) \Delta)-2 \sum_{i=1}^{n}\left[\cos ^{-1}\left(\frac{i-1}{n+1}\right)-\cos ^{-1}\left(\frac{i}{n+1}\right)\right] f(i \Delta) i \Delta\right) \\
& \times\left[2(n+1) \Delta \cos ^{-1}\left(\frac{n}{n+1}\right)\right]^{-1}
\end{aligned}
$$


valid for $0 \leqslant n \leqslant N$; note $t=n \Delta$ and $T=N \Delta$.

Numerical results of running this algorithm can be summarized as follows:

(1) For $\Delta=0.01$ (i.e. $N=100$ ), the algorithm worked very well. The maximum difference between the actual and reconstructed values of $f(x)$ was 0.0136 at $|x|=1$; a more typical difference was 0.0079 at $|x|=0.5$. No plot is given, as the actual and reconstructed values cannot be distinguished on it.

(2) Recall that $f(x)$ must be already known inside a circle of radius $\epsilon$, to initialize the invariant imbedding recursion. For this example, the minimum value of $\epsilon$ was found to be $\Delta$; specifying $f(x)$ at locations other than the origin did not improve the reconstruction.

(3) Decreasing $\Delta$ had little effect on the accuracy of the reconstruction. For example, $\Delta=0.005(N=200)$ resulted in differences of 0.0109 at $|x|=0.5$ and 0.0154 at $|x|=1$. The improved accuracy in modelling the continuous function $f(x)$ seems to be offset by roundoff error; note that division by $2(n+1) \Delta \cos ^{-1}(n /(n+1))$ is required in (3.3). Roundoff error can be ameliorated somewhat by precomputing the coefficient of $f(i \Delta)$ as

$$
\left(\frac{i}{n+1}\right)\left[\cos ^{-1}\left(\frac{i-1}{n+1}\right)-\cos ^{-1}\left(\frac{i}{n+1}\right)\right]\left[\cos ^{-1}\left(\frac{n}{n+1}\right)\right]^{-1}
$$

but roundoff error may still be a problem with this algorithm.

If $f(x)$ is not circularly symmetric, difficulties will arise near the origin, since $\Delta \theta$ is no longer small and the assumption that $f(x)$ is constant in a wedge subtending angle $\Delta \theta$ is no longer valid. There are two ways around this difficulty. One way is to make $\epsilon$, the radius of the circle in which $f(x)$ is assumed to be already known, large enough so that $\Delta \theta$ at radius $\epsilon$ is small enough for $f(x)$ to be assumed constant in the wedge subtending angle $\Delta \theta$. The recursion is then initialized at radius $|x|=\epsilon+\Delta$. The other way is explained next.

\subsection{Invariant imbedding algorithm for bandlimited functions}

If $f(x)$ is assumed to be angularly bandlimited, as discussed in subsection 2.2 , the following changes can be made in the invariant imbedding algorithm. Let $F_{m}(|x|)$ defined in (2.7a) be zero for $|m|>M$, for some $M$. Then both $f(|x|, \theta)$ and $\hat{f}(t, \phi)$ can be sampled in their angular variables at integer multiples of $2 \pi /(2 M+1)$ with no loss of information, since the samples may be interpolated to give the original functions. Suppose the data consist of the angularly-sampled 2D spherical means $\left\{\hat{f}\left(t, \phi_{k}\right), \phi_{k}=2 \pi k /(2 M+1), 0 \leqslant k \leqslant 2 M\right\}$. Also suppose $f(|x|, \theta)$ can be assumed to be piecewise constant in $|x|$, changing only at integer multiples of $\Delta$. Writing (3.1) for $\phi=\phi_{k}, 0 \leqslant k \leqslant 2 M$, substituting (2.6) in (3.1), and breaking up the integral in (3.1) into integrals over the annuli $\{n \Delta \leqslant|x| \leqslant(n+1) \Delta, 0 \leqslant n \leqslant N\}$ where $t=N \Delta$ yields the $2 M+1$ simultaneous linear equations in $2 M+1$ unknowns $F_{m}((N+1) \Delta)$

$$
\begin{aligned}
\hat{f}\left((N+1) \Delta, \phi_{k}\right)-\sum_{n=1}^{N} \sum_{m=-M}^{M} A_{m, n, k} F_{m}(n \Delta) & \\
=\sum_{m=-M}^{M} A_{m, N+1, k} F_{m}((N+1) \Delta) & 0 \leqslant k \leqslant 2 M
\end{aligned}
$$


$A_{m, n, k}=\frac{2 n \Delta}{m} \mathrm{e}^{\mathrm{i} m \phi_{k}}\left[\sin \left(m \cos ^{-1}\left(\frac{n-1}{N+1}\right)\right)-\sin \left(m \cos ^{-1}\left(\frac{n}{N+1}\right)\right)\right]$.

The linear system of equations (3.4) can be solved for the $F_{m}((N+1) \Delta)$, which can then be inserted into (2.6a) to give the reconstructed $f(|x|=t+\Delta, \theta)$. Note that setting $M=0$ in (3.4) and using the relation $\lim _{m \rightarrow 0} \sin (m x) / m \simeq x$ yields (3.3). However, the system of equations (3.4) becomes poorly-conditioned for large $m$; this is not surprising, for reasons explained in subsection 3.5 below.

\subsection{Geometric inversion algorithm for the causal spherical mean inverse problem}

Another approach to solving the causal spherical mean inverse problem is as follows. Given spherical means $\{\hat{f}(t, e), t<T\}$ of $\{f(x),|x|<T\}$, the values $\{f(x),|x|<T\}$ may be recovered from $\{\hat{f}(t, e), t<T\}$ as follows:

(1) Define $\hat{g}(t, e)=T^{n-1} \hat{f}(t, e)$ for $t<T$.

(2) Define $\hat{g}^{\prime}(t, e)=\hat{g}(T / t, e)$ for $t>1$.

(3) Compute $g^{\prime}\left(|x|, e_{x}\right)=\mathcal{R}_{(t, e) \rightarrow\left(|x|, e_{x}\right)}^{-1}\left\{\hat{g}^{\prime}(t, e)\right\}$ for $t>1$ and $|x|>1$.

(4) Define $g\left(|x|, e_{x}\right)=g^{\prime}\left(T /|x|, e_{x}\right)$ for $|x|<T$.

(5) Then $f(x)=g\left(|x|, e_{x}\right) /|x|^{2(n-1)}$ for $|x|<T$.

Note here that primes denote quantities that have been inverted across a sphere, and hats denote quantities that are transforms of unhatted quantities.

To prove this result we need the following lemma:

Lemme. Let $g(r)$ he any conntinuous function on $\mathcal{R}^{n}$, and consider the inversion of the Radon transform of its inversion $\left\{\mathcal{R}_{\left(|x|, e_{x}\right) \rightarrow(t, e)}\left\{g\left(T /|x|, e_{x}\right)\right\}_{t \rightarrow T / t}\right\}$. Then

$\left\{\mathcal{R}_{\left(|x|, e_{x}\right) \rightarrow(t, e)}\left\{g\left(T /|x|, e_{x}\right)\right\}_{t \rightarrow T / t}\right\}=t T^{n-1} \mathcal{B}_{\left(|x|, e_{x}\right) \rightarrow(t, e)}\left\{g\left(|x|, e_{x}\right) /|x|^{n}\right\}$.

Proof. We have

$$
\begin{aligned}
\left\{\mathcal{R}_{\left(|x|, e_{x}\right) \rightarrow(t, e)}\left\{g\left(T /|x|, e_{x}\right)\right\}_{t \rightarrow T / t}\right\} & \\
& =\int_{0}^{\infty} \int_{S} g\left(T /|x|, e_{x}\right) \delta\left(T / t-|x| e \cdot e_{x}\right)|x|^{n-1} \mathrm{~d} e_{x} \mathrm{~d}|x| \\
& =\int_{0}^{\infty} \int_{S} g\left(|x|, e_{x}\right) \delta\left(\frac{T}{t}-\frac{T}{|x|}\left(e \cdot e_{x}\right)\right) \frac{T^{n}}{|x|^{n+1}} \mathrm{~d} e_{x} \mathrm{~d}|x| \\
& =\int_{0}^{\infty} \int_{S} g\left(|x|, e_{x}\right) \delta\left(|x|-t e \cdot e_{x}\right) \frac{t T^{n-1}}{|x|^{n}} \mathrm{~d} e_{x} \mathrm{~d}|x| \\
& =t T^{n-1} \int_{S} g\left(|x|=t e \cdot e_{x}, e_{x}\right) /|x|^{n} \mathrm{~d} e_{x} \\
& =t T^{n-1} \mathcal{B}_{\left(|x|, e_{x}\right) \rightarrow(t, e)}\left\{g\left(|x|, e_{x}\right) /|x|^{n}\right\} .
\end{aligned}
$$

In (3.6) we changed variables from $|x|$ to $T /|x|$ and used the scale property $\delta\left(\frac{y T}{|x| t}\right)=\frac{|x| t}{T} \delta(y)$ of the impulse.

The algorithm follows immediately from the lemma and (2.4) by setting

$$
\begin{aligned}
& f(x)=g\left(|x|, e_{x}\right) /|x|^{2(n-1)} \\
& \hat{f}(t, e) T^{n-1}=\left\{\mathcal{R}_{\left(|x|, e_{x}\right) \rightarrow(t, e)}\left\{g\left(T /|x|, e_{x}\right)\right\}_{t \rightarrow T / t}\right\} .
\end{aligned}
$$

Note that step 3 is the regularized exterior Radon transform problem (we only require $g^{\prime}\left(|x|, e_{x}\right)$ for $\left.|x|>1\right)$, which can be solved as discussed in subsection 2.2 . 


\subsection{Comments}

The form of the algorithm in subsection 3.4 is not surprising: as shown in subsection 1.4, geometrically inverting a plane across the sphere of radius $T$ results in a sphere which passes through the origin, and vice versa. This explains why the inversions $t \rightarrow T / t$ and $|x| \rightarrow T /|x|$ transform a problem involving integrals over spheres into a problem involving integrals over planes.

Note that the inverse Radon transform in step 3 could be performed by a filtered backprojection operation (2.9), if not for the restrictions on $|x|$ and $t$. This is not surprising; previous work on inversion of spherical means has generally involved backprojection over spheres, or equivalent operations $[1,2]$. The difference between previous work and the above algorithm is that the causality restrictions imposed on the problem preclude the use of backprojection to compute $\mathcal{R}^{-1}$ in step (3).

The inverse Radon transform in step (3) must be performed anti-causally, computing $\left\{g^{\prime}\left(|x|, e_{x}\right),|x|>1\right\}$ from $\left\{\hat{g}^{\prime}(t, e), t>1\right\}$; this is the regularized exterior Radon transform problem described in subsection 2.2 . Note that since we only require $g^{\prime}\left(|x|, e_{x}\right)$ for $|x|>1$ (i.e. $t=1$ in section 2.2 ), the problem is not ill-conditioned; however, it will be poorly conditioned (see the end of subsection 2.2).

We also require that $g^{\prime}\left(|x|, e_{x}\right)$ be known to equal zero for $|x|>R$ for some arbitrarily large $R$; this is equivalent to constraining $f\left(|x|, e_{x}\right)=0$ for $x$ inside the small sphere $|x|<\epsilon$, where $\epsilon=T / R$ (this also avoids difliculties with $g\left(|x|, e_{x}\right) /|x|^{n}$ increasing without bound at the origin). Thus once again we see that knowledge of $f(x)$ inside a small sphere is necessary for $\{f(x),|x|<T\}$ to be uniquely determined from $\{\hat{f}(t, e), t<T\}$.

Specifically, suppose we wish to reconstruct $\{f(x),|x|<T\}$ from its 2D spherical means $\{\hat{f}(t, \phi), t<T\}$, and that $f(x)$ is known to be zero for $|x|<\epsilon$. The worst case of evaluating the Chebyschev polynomial in (2.8) is then

$$
P_{M}(R / 1)=P_{M}(T / \epsilon) \simeq(1 / 2)(2 T / \epsilon)^{M}
$$

for large $M$ and $T / \epsilon$. Hence the larger the radius $\epsilon$ of the circle of already-known values of $f(x)$, relative to the radius $T$ of the circle inside which the inverse spherical mean problem is defined, the better the conditioning of the inverse problem will be. From a purely geometric viewpoint, the significance of the ratio $T / \epsilon$, as opposed to the individual values of $T$ and $\epsilon$, is clear.

In fact, it is only necessary that $f(x)$ be known inside the small sphere $|x|<\epsilon$; it need not be zero inside this small sphere. To see this, let $f(x)=f_{\text {ext }}(x)+f_{\text {int }}(x)$, where $f_{\text {ext }}(x)=0$ for $|x|<\epsilon$, and $f_{\text {int }}(x)=0$ for $|x|>\epsilon$ (outside the small sphere) and takes on the known values of $f(x)$ for $|x|<\epsilon$. Since $f_{\text {int }}(x)$ is known completely, its spherical means may be computed and subtracted from the given $\hat{f}(t, e)$, resulting in the spherical means $\hat{f}_{\text {ext }}(t, e)$ of $f_{\text {ext }}(x)$. The above algorithm is then used to determine $f_{\text {ext }}(x)$ from $\hat{f}_{\text {ext }}(t, e)$, and then $f(x)$ is completely determined.

\subsection{Geometric inversion algorithm for the entire spherical mean inverse problem}

Recall that the entire spherical mean inverse problem is to reconstruct $f(x)$ from $\{\hat{f}(t, e)\}$ for all $t$ and $e$. The procedure given in subsection 3.4 for the causal problem can also be applied to the entire problem, simply drop the modifying inequalities $t\langle T, t\rangle 1$, etc since the lemma did not require these. Note that a sphere of very large radius $t$ geometrically inverts to a plane passing very close to the origin; 
integrals over these planes are not used in the exterior Radon transform problem, but they are used in the complete Radon transform problem of reconstructing $f(x)$ from all of its integrals over hyperplanes.

Thus the entire spherical mean inverse problem is equivalent, through geometric inversion, to the complete Radon transform problem. This is significant, since the complete Radon transform problem is relatively well-conditioned, and it may be solved quite readily using filtered back-projection [7]. In contrast, the exterior Radon transform problem, to which the causal spherical mean inverse problem is equivalent through geometric inversion, is relatively poorly conditioned, and it is generally considered to be impractical using real-world data.

Also note that, unlike the dual filtered backprojection algorithm of subsection 2.3, we do not require the data $\hat{f}(t, e)$ for all $t$ to compute $f(x)$ for any $x$. Indeed, we do not require $\hat{f}(t, e)$ for $t>1 / \epsilon$ if $f(x)$ is known for $|x|<\epsilon$-we merely proceed as in subsection 3.5 above. This algorithm appears to be the most useful one of those proposed in the current paper, provided the necessary data are available.

\section{Nullspace of spherical means}

\subsection{Characterization of nullspace}

Without the restriction that $f(x)$ is known inside a small sphere, the modificd spherical mean inverse problem does not have a unique solution. That is, $\mathcal{B}$ has a non-empty nutlspace $\mathcal{N}$, where

$$
\mathcal{N}=\{f:(\mathcal{B} f)(t, c)=0 \text { for } t<T\}
$$

(recall the problem is only defined for $|x|<T$ and $t<T$ ). The non-uniqueness then follows since it is possible for two different functions $f_{1}(x)$ and $f_{2}(x)$ to havc the same set of spherical means for $t<T$; all that is needed is $f_{1}(x)-f_{2}(x) \in \mathcal{N}$. We now investigate the nullspace $\mathcal{N}$ of $\mathcal{B}$.

Let $f_{\text {ext }}(x)$ be any function such that $f_{\text {ext }}(x)=0$ for $|x|<T$, i.e. $f_{\text {ext }}(x)$ is a 'hollow' function. Then $\left(\mathcal{H} \frac{\mathrm{d}}{\mathrm{d} t}\right)^{n-1} \mathcal{R}_{\left(|x|, e_{x}\right) \rightarrow(t, e)}\left\{f_{\text {ext }}(x)\right\} \in \mathcal{N}$ by (2.9). Hence we can construct members of $\mathcal{N}$ by taking any hollow function and applying the operator $\left(\mathcal{H} \frac{\mathrm{d}}{\mathrm{d} t}\right)^{n-1} \mathcal{R}$ to it. This might seem like a large class of non-zero functions, but the following theorem shows that this class is not as large as it might seem.

Theorem. Let $f_{\text {ext }}(x)=0$ for $|x|<T$ so that $f_{\text {ext }}(x)$ is a hollow function. Compute (compare to $(2.10)$ )

$$
\begin{aligned}
& \check{f}_{\text {ext }}(t, e)=\mathcal{B}_{\left(|x|, e_{x}\right) \rightarrow(t, e)}^{-1}\left\{f_{\text {ext }}(x)\right\}=\left(\mathcal{H} \frac{\mathrm{d}}{\mathrm{d} t}\right)^{n-1} \mathcal{R}_{\left(|x|, e_{x}\right) \rightarrow(t, e)}\left\{f_{\text {ext }}(x)\right\} \\
& \check{f}_{\text {ext }}(t, e)=\mathcal{B}_{\left(|x|, e_{x}\right) \rightarrow(t, e)}^{-1}\left\{f_{\text {ext }}(x)\right\} \in \mathcal{N}
\end{aligned}
$$

Let $\epsilon>0$ be arbitrarily small. Then if $\check{f}_{\text {ext }}(t, e)=0$ for $t<\epsilon$, then $\check{f}_{\text {ext }}(t, e)=0$ for $t<T$, so that $\check{f}_{\text {ext }}(t, e)$ is a trivial member of $\mathcal{N}$ (zero everywhere in the region of interest $t<T)$. The significance of this theorem is that although $f_{\text {ext }}(x)$ is zero for $|x|<T,\left(\mathcal{H} \frac{d}{\mathrm{dt}}\right)^{n-1} \mathcal{R}\left\{f_{\text {ext }}(x)\right\}$ must be non-zero near the origin $t=0$ for it to be a non-trivial member of $\mathcal{N}$. Hence some choices of $f_{\text {ext }}(x)$ will give rise to trivial members of $\mathcal{N}$, so $\mathcal{N}$ is not as large as it seems. 
Proof 1. Using geometric reflection as in subsection 3.3, this result follows immediatcly from the uniqueness result of the exterior Radon transform (see subsection 2.2).

Proof 2. Exchange $\left(|x|, e_{x}\right)$ and $(t, e)$, and apply the lemma with

$$
\begin{aligned}
& g\left(|x|, e_{x}\right)=|x|^{n} \check{f}_{\text {ext }}\left(|x|, e_{x}\right) \\
& t T^{n-1} f_{\text {ext }}(t, e)=\left\{\mathcal{R}_{\left(|x|, e_{x}\right) \rightarrow(t, e)}\left\{g\left(T /|x|, e_{x}\right)\right\}_{t \rightarrow T / t}\right\} .
\end{aligned}
$$

Again the result follows from uniqueness of the exterior Radon transform.

Proof 3. From the definition (2.1) of the Radon transform and the fact that $f_{\text {ext }}\left(|x|, e_{x}\right)=0$ for $|x|<T$

$\check{f}_{\text {ext }}(t, e)=\int_{T}^{\infty} \int_{S} f_{\text {ext }}\left(|x|, e_{x}\right)\left(\mathcal{H} \frac{\mathrm{d}}{\mathrm{d} t}\right)^{n-1} \delta\left(t-|x| e \cdot e_{x}\right)|x|^{n-1} \mathrm{~d} e_{x} \mathrm{~d}|x|$.

Since $t<T<|x|$ always, the integrand will always have two singularities at $t /|x|=e \cdot e_{x}$. Since the integral is zero for all $t<\epsilon, f_{\text {ext }}\left(|x|, e_{x}\right)$ must equal zero at these two singularities, so we have $f_{\text {ext }}\left(|x|=t /\left(e \cdot e_{x}\right), e_{x}\right)=0$ for all $t<\epsilon$ and all $e$. Hence we may choose $e$ sufficiently close to orthogonal to $e_{x}$ to make $e \cdot e_{x}=\epsilon / t$ for any $t<T$. Then, since $f\left(\epsilon /\left(e \cdot e_{x}\right), e_{x}\right)=0$, we have $f\left(t, e_{x}\right)=0$ for any $t$ such that there are singularities, i.e. for any $t$ such that $t<T<|x|$.

\subsection{Example}

It may seem strange that while $\mathcal{R}\left\{f_{\text {ext }}(x)\right\}$ is clcarly non-zero for $t<T$, its filtration $\check{f}_{\text {ext }}(t, e)=\left(\mathcal{H} \frac{\mathrm{d}}{\mathrm{d} t}\right)^{n-1} \mathcal{R}\left\{f_{\text {ext }}(x)\right\}$ may be zero for $t<T$. To illustrate this, let $T=1$ and consider the 'hollow ball' (in $\mathcal{R}^{3}$ ) function $f_{\text {ext }}(x)=1$ if $1<|x|<2$ and $=0$ otherwise. Then

$$
\mathcal{R}_{\left(|x|, e_{x}\right) \rightarrow(t, e)}\left\{f_{\text {ext }}(x)\right\}= \begin{cases}0 & \text { if } t>2 \\ \pi\left(4-t^{2}\right) & \text { if } 1<t<2 \\ 3 \pi & \text { if } 0<t<1\end{cases}
$$

so that although $\mathcal{R}\left\{f_{\text {ext }}(x)\right\} \neq 0$ for $t<1, \check{f}_{\text {ext }}(t)=\frac{\mathrm{d}^{2}}{\mathrm{~d} t^{2}} \mathcal{R}\left\{f_{\text {ext }}(x)\right\}=0$ for $t<1$.

\section{Conclusion}

We have presented several procedures for reconstructing a function $f\left(|x|, e_{x}\right)$ from its spherical means $\hat{f}(t, e)$ on spheres that pass through the origin. If we are given the data $\hat{f}(t, e)$ for all sphere diameters $t$ (the entire problem), $f\left(|x|, e_{x}\right)$ may be reconstructed directly using the Radon transform and a derivative-Hilbert transform (see subsection 2.3). If we are given only $\{\hat{f}(t, e), 0 \leqslant t \leqslant T\}$ (the causal problem), we may reconstruct $\left\{f\left(|x|, e_{x}\right), 0 \leqslant|x| \leqslant T\right\}$ using invariant imbedding; a simple numerical example was given, and a variation for bandlimited functions $f\left(|x|, e_{x}\right)$ was also given (see subsections 3.1-3.3).

Both the entire and causal problems may also be solved by inverting the data across the sphere of radius $T$, performing a complete inverse Radon transform (for 
the entire problem) or an anti-causal or exterior inverse Radon transform (for the causal problem), and then inverting the result about the sphere of radius $T$. These geometric inversions map the spheres over which the function is integrated into planes, thus mapping the spherical mean transform problem into a Radon transform problem. Since the Radon transform problem is well understood, this geometric inversion approach would seem to be preferable. Note that the complete Radon transform problem is relatively well conditioned, while the exterior Radon transform problem is relatively poorly conditioned; similar remarks seem to apply to the entire and causal spherical mean problems, respectively.

The problem was shown to have a unique solution, provided that the function is specified on an arbitrarily small sphere centred on the origin; the nullspace of the spherical mean operator missing this specification was also investigated. One interesting possibility for further research would be to solve the exterior Radon transform problem by reflecting it into a spherical mean problem, and then applying the invariant imbedding algorithm to solve this problem. Note that invariant imbedding cannot be applied directly to the exterior Radon transform problem, since it would operate on the unbounded domain $|x|>t$.

\section{Acknowledgments}

The author would like to thank one of the reviewers for a careful reading of the first version of this paper. This research was supported by the Office of Naval Research under grant N00014-90-J-1897.

\section{References}

[1] John F 1955 Plane Waves and Spherical Means (New York: Wiley)

[2] Fawcett J A 1985 Inversion of $N$-dimensional spherical averages SIAM J. AppL Math. 45 336-41

[3] Cohen J and Bleistein F 1979 Velocity inversion procedure for acoustic waves Geophysics $441077-85$

[4] Norton S J and Linder M 1981 Ultrasonic reflectivity imaging in three dimensions: exact inverse scattering solutions for plane, cylindrical, and spherical apertures IEEE Trans. Biomed. Eng. BME-28 202-20

[5] Devaney A J 1984 Geophysical diffraction tomography IEEE Trans. Geosci. GE-22 3-13

[6] Cormack A M 1964 Representation of a function by its line integrals, with some radiological applications II J. Appl. Phys. 35 2908-13

[7] Deans S R 1983 The Radon Transform and Some of its Applications (New York: Wiley)

[8] Abramowitz M and Stegun I 1965 Handbook of Mathematical Functions (New York: Dover) 